\title{
PRN OPINION PAPER: Application of precision medicine across pharmacy specialty areas
}

Kelly E. Caudle Pharm.D., Ph.D. ${ }^{1}$ () | Roseann S. Gammal Pharm.D., ${ }^{1,2}$ () |

Jason H. Karnes Pharm.D., Ph.D. ${ }^{3}$ | Janna Afanasjeva Pharm.D. ${ }^{4}$ |

Keri C. Anderson Pharm.D. | Erin F. Barreto Pharm.D., M.Sc. ${ }^{5,6}$ |

Craig Beavers Pharm.D. ${ }^{7,8}$ | Shubha Bhat Pharm.D., M.S. ${ }^{9}$ (] |

Kara L. Birrer Pharm.D. ${ }^{10}$ | Elias B. Chahine Pharm.D., FCCP ${ }^{11}$ |

Christopher R. Ensor Pharm.D., FCCP ${ }^{12}$ (1) | Stephanie A. Flowers Pharm.D., Ph.D. ${ }^{13}$ @ |

Christine M. Formea Pharm.D., FCCP ${ }^{14}$ | Jomy M. George Pharm.D. ${ }^{15}$ |

Rena A. Gosser Pharm.D. ${ }^{16}$ (1) | Mary F. Hebert Pharm.D., FCCP ${ }^{17}$ |

Lamis R. Karaoui Pharm.D. ${ }^{18}$ (] | Jimmi Hatton Kolpek Pharm.D. ${ }^{8}$ |

James C. Lee Pharm.D. ${ }^{13}$ | Jonathan G. Leung Pharm.D. ${ }^{14}$ । |

Angela Q. Maldonado Pharm.D. ${ }^{19}$ ๑ | Molly G. Minze Pharm.D. ${ }^{20}$ |

Rebecca A. Pulk Pharm.D., M.S. ${ }^{21}$ | Chasity M. Shelton Pharm.D. ${ }^{22}$ |

Maria Sheridan Pharm.D. ${ }^{23}$ | Michael A. Smith Pharm.D. ${ }^{24}$ (1) |

Scott Soefje Pharm.D., MBA, FCCP ${ }^{14}$ | Eglis Tellez-Corrales Pharm.D. ${ }^{25}$ |

Christine M. Walko Pharm.D., FCCP ${ }^{26,27}$ | Larisa H. Cavallari Pharm.D. ${ }^{28}$

\footnotetext{
${ }^{1}$ Department of Pharmaceutical Sciences, St. Jude Children's Research Hospital, Memphis, Tennessee

${ }^{2}$ Department of Pharmacy Practice, MCPHS University School of Pharmacy, Boston, Massachusetts

${ }^{3}$ Department of Pharmacy Practice and Science, University of Arizona College of Pharmacy, Tucson, Arizona

${ }^{4}$ Drug Information Group, University of Illinois College of Pharmacy, Chicago, Illinois

${ }^{5}$ Department of Pharmacy, Mayo Clinic, Rochester, Minnesota

${ }^{6}$ Robert D. and Patricia E. Kern Center for the Science of Health Care Delivery, Mayo Clinic, Rochester, Minnesota

${ }^{7}$ Department of Pharmacy Service, University of Kentucky Healthcare, Lexington, Kentucky

${ }^{8}$ Department of Pharmacy Practice \& Science, University of Kentucky College of Pharmacy, Lexington, Kentucky

${ }^{9}$ Department of Pharmacy, Boston Medical Center, Boston, Massachusetts

${ }^{10}$ Pharmacy Services, Orlando Regional Medical Center/Orlando Health, Orlando, Florida

${ }^{11}$ Department of Pharmacy Practice, Palm Beach Atlantic University Lloyd L. Gregory School of Pharmacy, West Palm Beach, Florida

${ }^{12}$ Department of Pharmacy, AdventHealth Orlando, Orlando, Florida

${ }^{13}$ Department of Pharmacy Practice, University of Illinois at Chicago, Chicago, Illinois

${ }^{14}$ Department of Pharmacy Services, Mayo Clinic, Rochester, Minnesota

${ }^{15}$ Clinical Pharmacokinetics Research Unit, Clinical Center Pharmacy, National Institutes of Health, Bethesda, Maryland

${ }^{16}$ Department of Pharmacy, University of Washington Medicine, Seattle, Washington

${ }^{17}$ Departments of Pharmacy and Obstetrics \& Gynecology, University of Washington, Seattle, Washington

${ }^{18}$ Department of Pharmacy Practice, Lebanese American University School of Pharmacy, Byblos, Lebanon
} 
${ }^{19}$ Department of Transplant Surgery, Vidant Medical Center, Greenville, North Carolina

${ }^{20}$ Department of Pharmacy Practice, Texas Tech University Health Sciences Center School of Pharmacy, Abilene, Texas

${ }^{21}$ Corporate Pharmacy Services, Yale New Haven Health, New Haven, Connecticut

${ }^{22}$ Department of Clinical Pharmacy and Translational Science, The University of Tennessee Health Science Center, Memphis, Tennessee

${ }^{23}$ Fresenius Kabi USA, LLC, Lake Zurich, Illinois

${ }^{24}$ Department of Clinical Pharmacy, University of Michigan, Ann Arbor, Michigan

${ }^{25}$ Department Pharmacy Practice, College of Pharmacy, Marshall B Ketchum University, Fullerton, California

${ }^{26}$ DeBartolo Family Personalized Medicine Institute, Moffitt Cancer Center, Tampa, Florida

${ }^{27}$ Department of Oncologic Sciences, Morsani College of Medicine, University of South Florida, Tampa, Florida

${ }^{28}$ Department of Pharmacotherapy and Translational Research and Center for Pharmacogenomics, University of Florida, Gainesville, Florida

\section{Correspondence}

Kelly E. Caudle, Department of Pharmaceutical Sciences, St. Jude Children's Research Hospital, 262 Danny Thomas PI, Memphis, TN 38105.

Email: kelly.caudle@stjude.org
Clinical pharmacists have been incorporating precision medicine into practice for decades. Drug selection and dosing based on patient-specific clinical factors such as age, weight, renal function, drug interactions, plasma drug concentrations, and diet are expected as part of routine clinical practice. Newer concepts of precision medicine such as pharmacogenomics have recently been implemented into clinical care, while other concepts such as epigenetics and pharmacomicrobiomics still predominantly exist in the research area but clinical translation is expected in the future. The purpose of this paper is to describe current and emerging aspects of precision medicine as it relates to clinical pharmacy across a variety of specialty areas of practice, with perspectives from the American College of Clinical Pharmacy Practice and Research Network membership.

\section{KEYWORDS}

pharmacogenomics, pharmacotherapy, precision medicine

\section{1 | INTRODUCTION}

The term, "precision medicine" has infiltrated the national dialog about the future of health care, but misconceptions exist as to the exact meaning of the phrase. Precision medicine transcends genomics, incorporating all known data-including variability in genes, environment, and lifestyle-to target specific treatments and/or disease prevention strategies for each patient. ${ }^{1}$ The goal is simple: ensure that each patient is receiving the right drug at the right dose at the right time. Many terms have been used to describe this concept or variations of it, including personalized medicine, individualized medicine, and more recently, precision pharmacotherapy. ${ }^{2}$ The term precision medicine may be preferred to personalized or individualized medicine because the concept does not entail developing drugs and medical devices unique to every patient. Rather, the concept seeks to classify individuals into subpopulations that differ in their response to treatment and susceptibility to disease. It is also important to note that "precision" is being used in a colloquial sense that encompasses both accuracy and precision, in contrast to the precision (reproducibility) of a measurement system.

While the term "precision medicine" is relatively new, the concept itself is not new to the pharmacy profession as pharmacists have been incorporating precision medicine into practice for decades. Drug selection and dosing based on patient-specific clinical factors such as age, weight, renal function, drug interactions, plasma drug concentrations, and diet are expected as part of routine clinical practice. Aspects of precision medicine, such as pharmacogenomics, are increasingly applied to patient care, and pharmacists are wellsuited to play a key role in advancing these efforts. The Clinical Pharmacogenetics Implementation Consortium (CPIC) guidelines provide clinicians with evidence-based information about how to translate pharmacogenomic data into prescribing decisions. Gene/drug pairs with published guidelines have a high level of evidence for implementation (PharmGKB levels 1A/B). Other areas, including pharmacomicrobiomics, epigenetics, and metabolomics (Table 1), still predominantly exist in the research sphere but clinical translation is expected in the future.

The purpose of this paper is to discuss current and emerging aspects of precision medicine as it relates to clinical pharmacy across a variety of specialty areas of practice, with perspectives from across the American College of Clinical Pharmacy (ACCP) Practice and Research Network (PRN) membership. The writing of this paper was led by the Pharmacokinetics/Pharmacodynamics/Pharmacogenomics PRN but represents the opinion of many of the ACCP PRNs (see sections below). It does not necessarily represent an official ACCP commentary, guideline, or statement of policy or position. 


\section{2 | PERSPECTIVES ON PRECISION MEDICINE BY PRN}

\section{1 | Disease-specific specialties}

\subsection{1 | Cardiology PRN}

Within the field of cardiovascular medicine, examples of individualized therapy include selection of antihypertensive therapy based on the patient's comorbid conditions (eg, prescribing an angiotensin converting enzyme inhibitor for nephro-protection in a patient with renal disease $^{9}$ and adding hydralazine/isosorbide to heart failure therapy for patients of African ancestry with continued symptoms ${ }^{10}$ ). Data analytics and advanced statistical techniques have allowed investigators to determine comorbidities or patient-specific variables that increase the risk for important clinical events, such as ischemia or bleeding, and assign a weighted score to each comorbidity/variable. These scoring systems, such as the Dual Antiplatelet Therapy score or the CathPCI bleeding risk score, assist the provider in selecting the type and duration of antiplatelet therapy. ${ }^{11,12}$ Cardiovascular practitioners also commonly individualize warfarin based on clinical factors such as age, weight, diet, and concomitant therapy. ${ }^{13}$

Genotype provides additional guidance to maximize cardiovascular drug safety and efficacy. Data are strongest for CYP2C19 and clopidogrel; CYP2C9 and VKORC1 for warfarin; and SLCO1B1 and simvastatin (Table 2). Pharmacogenomic algorithms are available to calculate warfarin dose requirements based on genotype and clinical factors (eg, warfarindosing.org). A number of institutions across the United States have clinically implemented genotyping into practice to assist with selection of $\mathrm{P} 2 \mathrm{Y} 12$ receptor antagonist after percutaneous coronary intervention, warfarin dosing, and statin prescribing. Data supporting improved outcomes with genotype-guided approaches are starting to emerge from pragmatic clinical trials and real-world studies. $^{14,15}$

\subsection{2 | Central nervous system PRN}

Psychiatric clinicians commonly employ patient-specific factors (eg, demographics, comorbidities, and symptoms), patient preference, and family history of response to guide medication selection. However, with the exception of lithium, there are little data to support many of these strategies. ${ }^{16}$ In reality, the overarching principle in psychiatry for medication selection is largely trial-and-error. Other factors influencing medication response include ensuring the correct diagnosis is made, appropriate dose titration, patient acceptance/understanding of illness, influence of stigma on taking medications,

TAB LE 1 Use of precision medicine in clinical practice

\begin{tabular}{|c|c|c|c|}
\hline Aspect & Definition & $\begin{array}{l}\text { Use in clinical } \\
\text { practice }\end{array}$ & Example(s) \\
\hline $\begin{array}{l}\text { Therapeutic drug } \\
\text { monitoring }\end{array}$ & $\begin{array}{l}\text { Measurement of medication concentrations in blood to } \\
\text { improve efficacy, reduce adverse events, or assess } \\
\text { adherence }\end{array}$ & Common & $\begin{array}{l}\text { Nortriptyline level } \\
\text { Vancomycin trough } \\
\text { Phenytoin level } \\
\text { PT/INR } \\
\text { Anti-Xa } \\
\text { Aminoglycosides peaks } \\
\text { and troughs }\end{array}$ \\
\hline Pharmacogenomics & $\begin{array}{l}\text { The study of how genes affect an individual's response } \\
\text { to medications (ie, efficacy and/or toxicity) Germline } \\
\text { variant: a DNA alteration (compared with wildtype) } \\
\text { contained within the germline passed from parent to } \\
\text { offspring (Table } 2 \text { ) Somatic variant: an alteration in } \\
\text { DNA that occurs after conception (Table 3) }\end{array}$ & $\begin{array}{l}\text { Occasional (common } \\
\text { in oncology) }\end{array}$ & $\begin{array}{l}\text { CYP450 testing } \\
H L A-B^{*} 57: 01 \text { testing }\end{array}$ \\
\hline $\begin{array}{l}\text { Nonpharmacogenomic } \\
\text { Biomarkers }\end{array}$ & $\begin{array}{l}\text { A measurable substance, structure or process in the } \\
\text { body or its products whose presence is indicative of } \\
\text { outcome or disease. }\end{array}$ & Occasional & Cystatin-C \\
\hline Epigenetics & $\begin{array}{l}\text { The natural occurrence of changes in gene expression } \\
\text { rather than changes in the DNA sequence }\end{array}$ & $\begin{array}{l}\text { Not currently used/ } \\
\text { research-based }\end{array}$ & \\
\hline $\begin{array}{l}\text { Microbiome } \\
\text { (Pharmacomicrobiomics) }\end{array}$ & $\begin{array}{l}\text { "The ecological community of commensal, symbiotic, } \\
\text { and pathogenic microorganisms" that inhabit our } \\
\text { bodies }{ }^{8} \text {; the microbiome composition contributes to } \\
\text { how individuals respond to medication leading to } \\
\text { suboptimal treatment }\end{array}$ & $\begin{array}{l}\text { Not currently used/ } \\
\text { research-based }\end{array}$ & $\begin{array}{l}\text { Over } 60 \text { drugs have been identified } \\
\text { to have microbiome interactions } \\
\text { according to the Pharmaco } \\
\text { Microbiomics database (www. } \\
\text { pharmacomicrobiomics.com) }\end{array}$ \\
\hline $\begin{array}{l}\text { Demographics/lifestyle/ } \\
\text { patient factors (weight, } \\
\text { age, race, environment, } \\
\text { diet, renal/liver function) }\end{array}$ & & Common & $\begin{array}{l}\text { Dose adjustment } \\
\text { Drug selection }\end{array}$ \\
\hline
\end{tabular}

Abbreviations: INR, international normalized ratio; PT, prothrombin time. 
TAB LE 2 Drugs with pharmacogenomic (PGx) guidance

\begin{tabular}{|c|c|c|c|}
\hline Drug(s) or drug class & PGx considerations & PGx guideline & $\begin{array}{l}\text { Other factors affecting drug response } \\
\text { that should be considered }\end{array}$ \\
\hline Abacavir & $H L A-B$ & CPIC, DPWG & HIV genotype \\
\hline \multirow[t]{2}{*}{ Atazanavir } & \multirow[t]{2}{*}{ UGT1A1 } & \multirow[t]{2}{*}{ CPIC } & Drug interactions \\
\hline & & & HIV-1 genotype \\
\hline Carbamazepine & \multicolumn{2}{|l|}{ HLA-A } & Concomitant therapy \\
\hline Celecoxib & CYP2C9 & $\mathrm{CPIC}^{\mathrm{a}}$ & $\begin{array}{l}\text { Renal function } \\
\text { Liver function }\end{array}$ \\
\hline Clopidogrel & CYP2C19 & CPIC, DPWG & $\begin{array}{l}\text { Disease history } \\
\text { Stent type } \\
\text { Bleeding risk }\end{array}$ \\
\hline Efavirenz & CYP2B6 & $\mathrm{CPIC}^{\mathrm{a}}$ & $\begin{array}{l}\text { Drug interactions } \\
\text { HIV-1 genotype } \\
\text { History of psychiatric comorbidities }\end{array}$ \\
\hline 5-Fluorouracil & DPYD & CPIC, DPWG & Myelosuppression \\
\hline Inhaled anesthetics & RYR1 & CPIC & Family history comorbidities \\
\hline Interferon alpha & INFL3 & CPIC & HCV type \\
\hline Irinotecan & UGT1A1 & DPWG, RNPGx/GPCO-Unicancer & Race \\
\hline Phenytoin & $\begin{array}{l}\text { CYP2C9 } \\
\text { HLA }\end{array}$ & CPIC, DPWG & $\begin{array}{l}\text { Drug interactions } \\
\text { Concomitant therapy }\end{array}$ \\
\hline Proton pump inhibitors & CYP2C19 & $\mathrm{CPIC}^{\mathrm{a}}$, DPWG & $\begin{array}{l}\text { Clinical factors } \\
\text { Concomitant therapy }\end{array}$ \\
\hline $\begin{array}{l}\text { Psychotropic agents } \\
\text { (eg, antidepressants, } \\
\text { antipsychotics) }\end{array}$ & $\begin{array}{l}\text { CYP2C19 } \\
\text { CYP2D6 }\end{array}$ & CPIC, DPWG & $\begin{array}{l}\text { Clinical factors } \\
\text { Psychiatric comorbidities } \\
\text { Accurate diagnosis } \\
\text { Drug interactions } \\
\text { Family history of drug response } \\
\text { Social factors }\end{array}$ \\
\hline Rasburicase & G6PD & CPIC & $\begin{array}{l}\text { Race } \\
\text { Pregnancy status } \\
\text { Food choices } \\
\text { Medication exposure }\end{array}$ \\
\hline Simvastatin & SLCO1B1 & CPIC & Concomitant therapy \\
\hline Tacrolimus & CYP3A5 & CPIC, DPWG & TDM \\
\hline Tamoxifen & CYP2D6 & CPIC, DPWG & Adherence \\
\hline Voriconazole & CYP2C19 & CPIC & $\begin{array}{l}\text { TDM } \\
\text { Drug interactions }\end{array}$ \\
\hline
\end{tabular}


TABLE 2 (Continued)

\begin{tabular}{|c|c|c|c|}
\hline Drug(s) or drug class & PGx considerations & PGx guideline & $\begin{array}{l}\text { Other factors affecting drug response } \\
\text { that should be considered }\end{array}$ \\
\hline Warfarin & $\begin{array}{l}\text { CYP2C9 } \\
\text { VKORC1 } \\
\text { CYP4F2 }\end{array}$ & CPIC & $\begin{array}{l}\text { Clinical factors } \\
\text { Social factors } \\
\text { Dietary considerations } \\
\text { Drug interactions } \\
\text { CPIC, CPNDS }\end{array}$ \\
\hline
\end{tabular}

Abbreviations: ACR, American College of Rheumatology ${ }^{3}$; CPIC, Clinical Pharmacogenetics Implementation Consortium ${ }^{4}$; CPNDS, Canadian Pharmacogenomics Network for Drug Safety ${ }^{5}$; DPGW, Dutch Pharmacogenetic Working Group ${ }^{6}$; HCV, hepatitis C virus; HIV, human immunodeficiency virus; RNPGx/GPCO Unicancer, French joint working group comprised of the National Pharmacogenetics Network [RNPGx] and the Group of Clinical Onco-pharmacology (GPCO-Unicancer) ${ }^{7}$; TDM, therapeutic drug monitoring.

${ }^{\mathrm{a} G u i d e l i n e ~ i n ~ p r o g r e s s . ~}$

TAB LE 3 Clinical examples of genotype-guided cancer chemotherapy

\begin{tabular}{lll} 
Somatic variant examples & & \\
\hline Drug target & Drug(s) & Common malignancy \\
$\begin{array}{l}\text { Echinoderm Microtubule Associated Protein-Like 4 and } \\
\text { Anaplastic Lymphoma Kinase (EML4-ALK) }\end{array}$ & Crizotinib, alectinib, ceritinib, brigatinib & Nonsmall cell lung cancer \\
BCR-ABL & Dasatinib, imatinib, nilotinib, bosutinib, ponatinib & Chronic myelogenous leukemia \\
BRAF & Vemurafenib, dabrafenib & Melanoma \\
MEK & Cobimetinib, trametinib & Melanoma \\
Epidermal growth factor receptor (EGFR) & Erlotinib, afatinib, gefitinib, osimertinib & Nonsmall cell lung cancer \\
Human epidermal growth factor receptor 2 (HER2) & Trastuzumab, lapatinib, pertuzumab, & Breast cancer, gastric cancer \\
Janus kinase 2 (JAK2) & Ado-trastuzumab emtansine & Myelofibrosis \\
Rearranged during transfection (RET) & Ruxolitinib & Medullary thyroid cancer \\
ROS1 & Vandetanib, cabozantinib & Nonsmall cell lung cancer
\end{tabular}

drug-interactions, social/environmental factors (eg, smoking-induction of CYP450 1A2), and resource (patient and community) availability.

Therapeutic drug monitoring is one objective method to help maximize the effectiveness and reduce adverse events for certain psychiatric medications (eg, clozapine, lithium, and nortriptyline). ${ }^{17}$ However, the evidence to support therapeutic drug monitoring is limited or absent for a majority of psychiatric medications. The use of pharmacogenomic testing has begun to be applied to psychiatry in clinical practice. Clinical studies assessing outcomes following pharmacogenomic testing (eg, CYP2D6, CYP2C19) have been conducted mostly in patients with major depressive disorder ${ }^{18}$ and indicate the potential to reduce drug side effects, which can impact adherence to treatment. However, the influence on efficacy or other outcome measures has been mixed. ${ }^{18}$ There is less evidence demonstrating benefit of pharmacogenomic testing in other psychiatric disease states, and there has been some concern that test results could be misleading and potentially harm patients by avoiding certain medications on the premise of poorly correlated studies. ${ }^{19}$ There is significant evidence linking the HLA-B*15:02 allele, which occurs predominately in individuals of Asian ancestry, to risk for severe cutaneous reactions (eg, Stevens-Johnson syndrome and toxic epidermal necrolysis) with carbamazepine, and testing is recommended in genetically at-risk persons prior to carbamazepine use. Similar data exist for phenytoin.

\subsection{3 | Critical care PRN}

The rapidly changing profile, acute organ dysfunction, and multimorbidity alters drug pharmacokinetic profiles and pharmacodynamic responses in critically ill patients. Using precision medicine to tailor medication dosing to dynamic end organ function and apply therapeutic drug monitoring using clinical response markers and drug levels ${ }^{20}$ is common in critical care. Also, in adult and pediatric patients, novel biomarkers such as procalcitonin, ferritin, $\mathrm{C}$-reactive protein, cell surface markers, interleukin-6, monocyte chemoattractant protein and MAS, have been tested as tools to enhance severity of illness scores (eg, APACHE) and disease models. Recently, distinct endophenotypes of acute respiratory distress syndrome and sepsis have been identified that are being used to enrich patient selection for targeted drug therapies in clinical trials. ${ }^{21-23}$

Many of the drugs noted in Table 2 are commonly used in the critical care setting but whether acute illness alters gene expression and the corresponding drug response is poorly studied. It is known that certain genetic polymorphisms may introduce adverse drug reaction 
risks unique to the critical care environment such as sedative, analgesic, and antiepileptic response in traumatic brain injuries. ${ }^{24}$ However, the role of genetic testing in the critically ill remains to be defined.

\subsection{4 | Endocrine and metabolism PRN}

Precision medicine in the realm of endocrinology is a growing area, which is timely given the patient-centered and individualized care recommended by current clinical guidelines. Current treatment for type 1 diabetes lacks genetic testing precision; however, weight-based dosing of insulin therapy is standard of care. ${ }^{25}$ Future precision medicine tools that will afford clinicians the opportunity to detect patients who may develop beta-cell loss resulting in hyperglycemia and those who will experience disease complications will be an enormous advancement in the treatment of diabetes. ${ }^{25}$

Genetic testing is useful in classifying single gene aberrations that cause diabetes (approximately $1 \%-2 \%$ of diabetes), such as the HNF1A variant in maturity-onset diabetes of the young and KCNJ11 and $A B C C 8$ variants in neonatal diabetes. In type 2 diabetes, genetic polymorphisms have been observed that influence glycemic response to metformin (eg, SLC22A1, SLC47A1, and ATM). ${ }^{26}$ However, application of these tests in the clinical setting is currently limited as the clinical utility of these tests remains uncertain.

\subsection{5 | Gastrointestinal/liver/nutrition PRN}

Nutrition support provided through enteral and parenteral nutrition is one of the most individualized, patient-specific therapies constituted of macronutrients, micronutrients, and fluid required to sustain life in those that cannot meet their needs orally. Pharmacists assist with calculating caloric provisions based on patient-specific factors or indirect calorimetry measurements and detect nutrient deficiencies through laboratory monitoring and clinical evaluation. Therapy is continually altered as the patient's clinical status changes and tailored to replete deficiencies or maintain adequate nutritional health.

Treatment for gastrointestinal diseases, such as inflammatory bowel disease (IBD), hepatitis C (HCV), and gastroesophageal reflux disease, is often guided by renal/hepatic function, concurrent and prior medication use, adherence, present symptoms, and genotype. Presently, for IBD, monitoring troughs of certain tumor necrosis factor (TNF)-alpha inhibitors to predict the likelihood of antidrug antibodies is useful to detect efficacy and minimize toxicity. Concomitant therapy with immunomodulators lowers TNF-alpha induced immunogenicity, but causes hematopoietic toxicity and warrants genotype or enzyme testing of thiopurine S-methyltransferase (TPMT) and nudix hydrolase (NUDT15) to determine toxicity risk with thiopurines use. ${ }^{27}$

Similarly, precision medicine is applied in treating HCV (Table 2). The emergence of direct activating antivirals has boosted cure rates and improved the proportion of patients treated, but requires assessment of prior treatment history, disease severity, contraindications with concomitant medications, existing comorbidities (human immunodeficiency virus [HIV] or hepatitis B), adherence, HCV genotype, and viral load prior to initiation. Testing for resistance of certain proteins (eg, NS5A) is warranted in specific situations (eg, initiation of certain regimens, treatment failure) and may further guide treatment selection and duration. ${ }^{28}$ Finally, precision medicine can be applied in patients treated with proton pump inhibitors (PPIs) for acid-related disorder. PPIs are metabolized by CYP2C19 and CYP3A4, and CYP2C19 poor metabolizers may be at increased risk for adverse effects while rapid and ultrarapid metabolizers are at risk for treatment failure, with the strongest data related to Helicobacter pylori eradication. Thus, CYP2C19 genotyping may be useful in personalizing PPI dosing, though it is not broadly utilized in practice likely due to a lack of knowledge of the association and how to use genotype to guide prescribing. However, evidence review and guidelines for the use of CYP2C19 testing for PPI therapy are forthcoming by CPIC. ${ }^{29}$

\subsection{6 | Hematology/oncology PRN}

Precision medicine has revolutionized the field of oncology and led to advances in both the development and use of therapies to target somatic (tumor) genetic alterations as well as to improve toxicity and efficacy outcomes by analyzing germline pharmacogenomic alterations. Integrating the knowledge of germline and somatic alterations into clinical practice is now standard of care at many centers and provides an opportunity for pharmacists practicing in oncology to optimize treatment and supportive care recommendations. ${ }^{30}$

The increased understanding of cancer biology in conjunction with advances in molecular technology has helped identify numerous common drivers of oncogenesis. As molecular targets have been identified, oncology drug development has produced numerous successful targeted agents with thousands more currently in the development pipeline. ${ }^{31}$ Genetic tumor testing is performed to provide both prognostic and predictive information to guide further patient care. For example, common predictive alterations include EGFR activating variants in nonsmall cell lung cancer that are associated with a response to EGFR inhibitors such as erlotinib, gefitinib, afatinib and osimertinib, and BRAF V600 activating variants in melanoma that are associated with response to inhibitors of BRAF (vemurafenib and dabrafenib) and MEK (cobimetinib and trametinib). ${ }^{32}$ Numerous other examples of predictive genetic alterations in oncology exist and selected examples are presented in Table 2. Genetic testing of tumors is now part of standard practice to help determine disease risk classification in myeloid malignancies, identify standard of care targeted therapy options as discussed above, or identify treatment options for patients who are diagnosed with less common malignancies such as Merkel cell carcinoma or certain subtypes of sarcoma. Tumor genetic testing frequently involves not just assessing the clinical importance of one or two genes but can include consideration of the interplay of numerous genes that may be altered in a tumor. Numerous databases are available to assist with clinically interpreting genetic variants from these reports. ${ }^{30}$ Molecular tumor boards (MTBs), multidisciplinary groups of oncologists, pathologists, medical geneticists, basic scientists, pharmacists, and other interprofessional care team members, synthesize the literature for the individual patient and his or her unique characteristics to ultimately produce a treatment plan. ${ }^{30}$ Given 
a pharmacist's background in cancer biology and pharmacology, the profession is well positioned to provide valuable recommendations at MTB meetings, and some interdisciplinary MTBs have been developed and are under the leadership of pharmacists. ${ }^{30,33}$

Germline pharmacogenomic testing is also used in standard oncology practice to help minimize toxicity while maximizing efficacy of cytotoxic chemotherapy agents. The importance of drug and dose selection in oncology is underscored by the narrow therapeutic index of many oncology agents. Examples of germline pharmacogenomic testing in oncology include TPMT and NUDT15 testing for patients receiving mercaptopurine and thioguanine, commonly used in acute lymphocytic leukemia regimens, and DPYD testing for patients receiving fluorouracil or capecitabine, commonly used in colorectal cancers as well as other solid tumors. ${ }^{34-36}$ Other examples of relevant germline pharmacogenomic genes associated with drugs (eg, tamoxifen, allopurinol, and rasburicase) commonly used in the oncology population are listed in Table 2.

\subsection{7 | HIV PRN}

The goals of precision medicine and antiretroviral (ARV) therapy are multifactorial and are both drug- and disease-related. Drug-related factors include optimization of regimen efficacy, minimization of drug toxicity, and prevention of ARV resistance. Disease-related goals include reducing overall morbidity and mortality by optimizing immunologic recovery and virologic suppression. Interpatient variability presents unique challenges for people living with HIV (PLWH). Contributing factors include comorbid conditions, opportunistic infections, drug-drug (including complementary alternative medicines) and/or drug-disease interactions, medication adherence, ARV pharmacokinetics/pharmacodynamics (PK/PD), pharmacogenomics, viral genetics, age, gender, and ethnicity. Many of these factors have helped inform actionable practices and implementation of precision medicine into the clinical care of PLWH, while others are still being investigated.

The CPIC and current ARV guidelines by the US Department of Health and Human Services provide several recommendations for individualization of therapy. ${ }^{37}$ In the Food and Drug Administration (FDA) boxed warning for abacavir, screening for the HLA-B*57:01 allele is recommended prior to starting abacavir-containing regimens, as this approach has been found to significantly decrease the risk of a hypersensitivity reaction. Prior to initiating ARVs or when assessing the presence of ARV-associated resistant variants during treatment failure, HIV viral genotyping and drug specific assays (eg, HIV-1 tropism) are recommended to tailor and optimize therapy. When constructing a regimen, coinfection with hepatitis $B$ must be considered to ensure inclusion of a tenofovir and emtricitabine/lamivudine backbone. Individualizing therapy is particularly important in the current population of PLWH as more than $50 \%$ are over the age of 50 and have multiple comorbid conditions (eg, hepatitis C, dyslipidemia, coagulopathy) where drug-drug interactions and overlapping toxicities must be managed. ${ }^{38}$ Ongoing investigations to further support precision medicine such as therapeutic drug monitoring, optimal PK/PD indices of $A R V s$, gender differences in tolerability, ARV tissue distribution for the prevention of HIV, novel drug delivery systems, and pharmacogenomic variants are still underway. Other applications of pharmacogenomics to ARV include UGT1A1-guided atazanavir use $^{39}$ and CYP2B6-guided efavirenz dosing, ${ }^{40}$ though these are not yet widely used in clinical practice, likely because these agents have fallen out of favor in the United States due to the availability of better tolerated medications, and testing is not readily available outside of the United States.

\subsection{8 | Immunology/transplantation PRN}

Although guidelines exist, what constitutes standard of care management in solid organ transplantation varies widely, particularly with the application of precision medicine. Precision medicine is utilized in all phases of transplantation (pretransplant, peri-operatively, and posttransplant) by a multidisciplinary team which includes physicians, pharmacists, pathologists, immunologists, nurses, social workers, and dieticians. To determine if a patient has low or high immunologic risk, factors including race/ethnicity, class I and class II human leukocyte antigen (HLA) donor mismatch, age, calculated panel reactive antibody, $\mathrm{ABO}$ blood type, and donor characteristics as quantified by the Kidney Donor Profile Index should be considered while genomics are utilized to determine the type and exposure of immunosuppression used for each specific transplant patient.

The precision medicine needs of a transplant recipient change over time with the continuing goal of balancing adequate immunosuppression to prevent allograft injury with the risks of infection, malignancy, and adverse side effects. Tacrolimus is the cornerstone of modern immunosuppressive therapy yet there are significant challenges in defining what constitutes "adequate" levels for each individual patient. Perhaps the most clinically relevant example of pharmacogenomics in solid organ transplantation is CYP3A5 testing to guide tacrolimus (TAC) dosing (Table 2). CYP3A5normal and intermediate metabolizers ("expressers") are expected to require a dose that is 1.5-2 times higher than standard dosing to achieve therapeutic plasma concentrations. $^{38}$ Currently, in most medical centers, CYP3A5 genotyping to guide TAC dosing is not standard practice and dosing is guided by therapeutic drug monitoring (TDM). In contrast to TDM, which must be done after a drug is started, genetic testing can be done a priori to guide initial TAC dosing, thus potentially reducing the time to achieve therapeutic drug levels. Testing may be of particular benefit in centers that serve a racially diverse population given the higher frequency of functional CYP3A5 alleles in persons of African ancestry. African American patients are more likely to be CYP3A5 normal and intermediate metabolizers; hence, higher doses are recommended in this patient population. ${ }^{41}$

\subsection{9 | Infectious diseases PRN}

Infectious diseases clinicians routinely use PK and PD principles to guide treatment decisions. It is a common practice at hospitals for pharmacists to streamline antimicrobial selection based on infection 
site, microbiology culture, and sensitivity reports and to adjust the dosing of antimicrobials based on infection site and renal function. The Infectious Diseases Society of America and the Society for Healthcare Epidemiology of America guidelines for implementing an antimicrobial stewardship program recommend antimicrobial dosing optimization and microbiology diagnostics integration as key strategies to improve patient outcomes while halting antimicrobial resistance, decreasing adverse effects, and controlling costs. ${ }^{42}$ The guidelines specifically recommend that hospitals implement pharmacokinetic monitoring and adjustment programs for aminoglycosides and vancomycin, which has become a standard of practice for hospital-based pharmacists. ${ }^{42}$ The guidelines also recommend that antimicrobial stewardship programs advocate for alternative dosing strategies for broad-spectrum beta-lactams, which is becoming a common hospital practice for piperacillin/tazobactam, ceftazidime, cefepime, and meropenem. ${ }^{42}$ In addition, the guidelines recommend development of stratified antibiograms with selective and cascade reporting of antibiotics and the use of rapid diagnostic testing (eg, MALDI-TOF, PNA FISH \pm MCA, PCR, serology, etc.) and procalcitonin to optimize antimicrobial use, which is becoming increasingly popular in academic medical centers. ${ }^{42}$ Many molecular rapid diagnostic testing are capable of detecting genes associated with antimicrobial resistance and the FISH/MCA system is capable of revealing phenotypic resistance patterns. ${ }^{43}$ Although guidelines exist for the use of CYP2C19 genotype to guide voriconazole use, ${ }^{44}$ the use of pharmacogenomics to guide antimicrobial drug selection and dosing is rarely used at present. This may be reflective of a lack of knowledge about genetic associations with voriconazole disposition or about the availability of genetic testing to optimize voriconazole dosing. Alternatively, establishing processes by which genotype information is available at the point of prescribing is a challenge, yet is ideal for drugs like voriconazole, especially for life-threatening invasive fungal infections where it is critical to efficiently attain therapeutic drug levels. The expected increase in preemptive pharmacogenomic testing models in the future will help to address this challenge.

\subsubsection{0 | Nephrology PRN}

Pharmacists have long been at the forefront of precision medicine, specializing in tailoring medication doses for patients with kidney disease (Table 1) and for those receiving dialysis. More recently, advances in molecular genetics have informed development of novel biomarkers associated with estimated glomerular filtration rate (eGFR), end stage renal disease, acute kidney injury (AKI), and chronic kidney disease progression. Also, molecular genetics has identified genes and variants associated with kidney disease that can provide opportunities for targeted therapies. ${ }^{45}$

Urinary biomarkers for early detection of AKI have always been an area of great interest and study. Furthermore, attention has been paid to more fully characterizing phenotypes of drug-induced $A K I$ to better establish causality. The International Serious Adverse Events Consortium recently convened a panel (including pharmacists) which proposed four phenotypes of drug-induced kidney disease based on clinical presentation: AKI, glomerular disorder, tubular disorder, and nephrolithiasis/crystalluria. This framework is valuable for evaluating drug toxicity and patients at risk for drug-induced AKI. ${ }^{46}$

Several new serum and urinary biomarkers suitable for use as an adjunct or alternative to creatinine for kidney assessment have been identified. In 2018, FDA's Predictive Safety Testing Consortium approved the use of six urinary biomarkers to aid in the detection of $\mathrm{AKI}$ in early phase clinical trials including clusterin, cystatin-C, kidney injury molecule-1, N-acetyl-beta-D-gluconsaminidase, neutrophil gelatinase-associated lipopcalin, and osteopontin. ${ }^{47}$ Less invasive methods, including tracking transdermal fluorescent compounds, will enable clinicians to more accurately estimate GFR and identify early changes in kidney function. ${ }^{48}$ Additionally, the use of electronic health records and predictive analytics when combined with AKI biomarkers could be used to flag patients in real time. ${ }^{49}$

\subsubsection{1 | Pain and palliative care PRN}

Assessing (and reassessing) each patient's risks and benefits with respect to symptom directed therapy is essential to maintain efficacy with minimal toxicity. The basics of symptom management include understanding the extent and timeline of the disease or injury, the magnitude of the illness, expected relief (or not), symptom severity and interference, and functional limitations. Pain-specific assessments should also include pain sensitivity, central sensitization characteristics, a complete biopsychosocial assessment, and risks of dependence, abuse, respiratory depression, and diversion. ${ }^{50}$ Assessing comorbid conditions and concomitant medications are vital to estimating the risk-to-benefit of drug therapy, particularly opioids. Safe and effective management of pain and associated symptoms (eg, anxiety/ depression, fatigue) requires in-depth patient assessment and individualized treatment approaches.

Precision medicine for management of pain, whether acute or chronic, may include the application of pharmacogenomic testing. A recent comprehensive review of opioid pharmacogenomics and clinical pain management describes genetic associations with opioid effectiveness and safety. ${ }^{51}$ The most evidence exists for the CYP2D6 genotype related to codeine and tramadol response (Table 2). Of particular concern is the risk for serious adverse effects including respiratory depression and even death with tramadol or codeine use in CYP2D6 ultra-rapid metabolizers. Poor metabolizers, on the other hand, may not attain pain relief from codeine or tramadol. Notably, a number of drugs inhibit the CYP2D6 enzyme and may phenotypically convert patients with a normal metabolizer genotype to the intermediate or poor metabolizer phenotype. Other opioids such as oxycodone and hydrocodone are metabolized by CYP2D6 but limited data exist on how to use this information clinically. ${ }^{51}$ Other potential applications of pharmacogenomic data for pain management include CYP2D6 and CYP2C19 testing for tricyclic antidepressants selection and dosing, ${ }^{52,53}$ and CYP2C9 testing for select NSAIDs ${ }^{54}$ (Table 2). 


\subsubsection{2 | Perioperative care PRN}

There are many examples of practices used to guide patient care throughout the continuum of the perioperative period. Preadmission testing allows for identification of patients with specific intraoperative risks (eg, difficult airway, intraoperative or postoperative ischemic events, personal or family history of malignant hyperthermia $[\mathrm{MH}]$, postoperative nausea/vomiting [PONV], etc.) for whom early recognition and therapy implementation or adjustment can minimize or mitigate potential complications. For example, in patients with a history of hypertension, guidelines recommend establishing target systolic blood pressure (SBP) or mean arterial pressure (MAP) goals based on the patient's resting blood pressure (BP) prior to surgery rather than using a standardized approach. ${ }^{55}$ This patient-specific baseline is then used as the target $\pm 10 \%$ SBP or MAP throughout surgery to decrease risk of ischemic injury (particularly neurologic or renal). A recent clinical trial found significantly less neurologic and renal injury when patients were maintained at their target BP rather than at a standard BP. ${ }^{56}$ Precision medicine is also seen in the implementation of enhanced recovery pathways, including continuous hemodynamic and oxygen delivery monitoring to mitigate organ ischemia through goal-directed fluid therapy and/or the addition of inotropes or vasopressors while minimizing fluid overload. ${ }^{57}$

Precision medicine is incorporated in the perioperative arena for three specific scenarios: prevention of $\mathrm{MH}, \mathrm{PONV}$, and surgical site infections. Preventing the life-threatening complication of $\mathrm{MH}$ in patients with a personal or family history of $\mathrm{MH}$ now incorporates not only clinical history and a muscle biopsy with an in vitro contracture test (IVCT) but also DNA testing (Table 2). Current guidelines recommend at risk patients undergo DNA screening first and only proceed to the biopsy and IVCT if no $\mathrm{MH}$ variant is detected. ${ }^{58}$ PONV occurs in $30 \%-50 \%$ of all patients undergoing surgery. Identifying patients at risk using validated scoring systems facilitates the development of a patient- and procedure-specific antiemetic regimen ${ }^{59}$ and the CYP2D6 genotype can affect ondansetron efficacy (Table 2). Third, the selection and dosing of antimicrobial surgical prophylaxis based on patientspecific factors (eg, allergies and weight) and surgery-specific factors including type, location, and duration is employed to decrease the risk for surgical site infections. ${ }^{60}$

\subsubsection{3 | Pulmonary PRN}

There are several disorders in pulmonary medicine for which precision medicine is practiced today, including but not limited to cystic fibrosis (CF), interstitial lung disease, asthma, alveolar proteinosis, alpha-1 antitrypsin deficiency-associated emphysema, primary and secondary immunodeficiencies, and others. Prior to 2011, therapy for CF was limited to supportive care; however, with the approval of ivacaftor (Table 2), the first CFTR potentiator that targets specific CFTR defects (eg, G551D), CF patients with these inherited variants have seen greater improvement in lung function. ${ }^{61}$ Since the introduction of ivacaftor, other CFTR modulators have been introduced targeting other CFTR defects resulting in improved outcomes for this patient population. ${ }^{61}$ The FDA has recently expanded approval of these modulators to include rare CFTR variants based on in vitro data, which will expand access to these targeted therapies for smaller subsets of patients who may benefit.

Precision medicine has provided substantial advances in the diagnosis and management of asthma over the past decade. Asthma is now recognized as a substantially heterogeneous disease with wide variation in response to conventional therapies. ${ }^{62}$ Multiple investigators have studied several therapies for specific phenotypes of severe asthma, particularly those with detectible elevations in eosinophil levels in the sputum, including those targeted at IgE, interleukin (IL) 5 receptor alpha (IL-5Ra), IL-4Ra, and IL-13. These therapies, including omalizumab (anti-IgE), dupilumab (anti-IL4), reslizumab and mepolizumab (anti-IL5), and lebrikizumab (anti-IL13) are intended for patients with severe asthma symptoms that are uncontrolled on maximal medical therapy or as a means to minimize high-dose corticosteroid exposure. ${ }^{63}$ Importantly, these therapies are only effective in the asthmatic patient with the respective phenotypic polymorphisms as described above. Thus, precision medicine testing, diagnostic, and treatment approaches are critical to best caring for patients with the most severe manifestations of asthma.

\section{2 | Generalist/special population specialties}

\subsection{1 | Pediatrics PRN}

There are unique challenges with the use of precision medicine in pediatrics. The pediatric patient population includes neonates weighing $500 \mathrm{~g}$ to adolescents weighing $300 \mathrm{~kg}$, and within this population there exists many developmental physiologic differences resulting in a widely heterogeneous population. ${ }^{64}$ Children are a vulnerable population and dosing recommendations are often extrapolated from existing adult data. Despite legislation to promote pediatric drug development, only two-thirds of completed pediatric trials have resulted in pediatric labeling. ${ }^{65}$ Additionally, dosage formulation manipulation for extemporaneous enteral formulations and drug dilutions for intravenous products are daily occurrences to create formulations necessary for drug delivery to this patient population, commonly without validated formulation or supporting data. ${ }^{64}$ Despite these challenges, there have been advances made towards precision medicine use in pediatrics.

Therapeutic drug monitoring (TDM) is an effective approach for precision medicine in pediatrics, including neonates and infants, specifically for medications with a narrow therapeutic range. ${ }^{65}$ TDM is most commonly used for vancomycin, aminoglycosides, digoxin, caffeine, and anticonvulsants. ${ }^{66}$ Using knowledge of developmental pharmacological differences across the age continuum and pharmacokinetic/pharmacodynamic drug information from adult data, a pediatric pharmacist can make dosing recommendations and then use TDM to assess the efficacy and/or safety of the dose. In the future, incorporating TDM into the drug development phase rather than relying on fixed-dose or strictly weight-based pediatric trials may facilitate better pediatric indication labeling. ${ }^{65}$ This may result in 
appropriate evidence-based medication selection and improved initial dosing recommendations for efficacy and safety with a reduced need for TDM.

Pharmacogenomic testing in pediatrics is evolving and expanding. Areas that are currently being studied include pain management, immunosuppression, childhood cancer, mental health, asthma, neurology, and cystic fibrosis. In pain management, CYP2D6 genotyping has been used to identify polymorphisms affecting codeine metabolism and increasing risk for toxicity or poor analgesic response (Table 2). Based on reports of toxicity including death with codeine use in children with the CYP2D6 ultra-rapid metabolizer phenotype, a boxed warning was added to the codeine labeling warning against codeine use in patients less than 18 years of age. ${ }^{67}$ Despite this, codeine remains the most commonly prescribed opioid in children less than 18 years of age highlighting the potential role for CYP2D6 testing when prescribing codeine. ${ }^{67}$ The use of precision medicine in immunosuppression, cancer, mental health, asthma, and cystic fibrosis are discussed in the respective sections of this paper.

Appreciating the effects of ontogeny during the drug development phase may also result in improved drug dosing, thereby increasing safety and decreasing toxicity profiles of medications given to pediatric patients. ${ }^{68}$

\subsection{2 | Women's health PRN}

Sex-related differences impact drug PKs and PDs and thus drug selection and dose. For example, antithrombotic agents (eg, aspirin, GPIlbllla inhibitors) that are used to treat cardiovascular disease have different pharmacokinetics in women, which may increase adverse effects and require dosage adjustments. ${ }^{69}$ Also, the FDA recommends that an initial dose for women of zolpidem be only $5 \mathrm{mg}$ because clearance of the drug is lower in women as compared with men. Women also have a higher risk of drug-induced long QT syndrome with antiarrhythmics (eg, quinidine, procainamide and disopyramide, sotalol and amiodarone) ${ }^{70}$ and antipsychotic drugs (eg, thioridazine, haloperidol, risperidone, olanzapine, and ziprasidone). ${ }^{71,72}$ In the treatment of depression, whereas men respond better to tricyclic antidepressants, women respond better to selective serotonin reuptake inhibitors. $^{73,74}$ Furthermore, the impact of oral contraceptives on pharmacokinetics and pharmacodynamics needs to be considered. Among other risks from oral contraceptives, women using these products may be at increased risk of venous thromboembolism. Patients with thrombophilias such as Factor $\mathrm{V}$ Leiden or prothrombin variant are predisposed to a particularly high risk of clotting, and safer alternative contraceptive measures should be considered. We have included a limited number of examples here; however, it is important to recognize that there are a broad range of sex- and gender-based medication issues that need to be taken into account in precision medicine.

Clinical pharmacists incorporate knowledge of pregnancy-induced changes in pharmacokinetics (eg, changes in renal filtration, renal drug transport, drug metabolism and binding) into practice to optimize efficacy at the same time as considering maternal, fetal, and neonatal safety in medication selection and dosage. For example, nifedipine, a CYP3A substrate, is known to have a higher apparent oral clearance and shorter half-life during pregnancy suggesting that higher doses and a shorter dosing interval should be considered. Another example is when using metoprolol for rate control of supraventricular tachycardias, a much higher dosage is needed during pregnancy. This dosage escalation not only reflects the increased elimination of metoprolol, but also the gestational age-dependent increase in heart rate that occurs in normal pregnancy. As an alternative to metoprolol, atenolol should be considered because it avoids the induction seen in the metabolism of metoprolol and provides a more predictable pharmacokinetic profile during pregnancy. Unfortunately, at many institutions, pregnant women are receiving exactly the same medications and doses as they received prior to pregnancy resulting in unnecessary subtherapeutic concentrations or toxic effects. Even more concerning is when all medications are discontinued because the woman becomes pregnant leading to a wide range of complications which are far more dangerous to the pregnant woman, fetus, and neonate than the continuation of those medications during pregnancy. Thus, precision medicine during pregnancy must include a risk-benefit analysis of the medications as well as risks of untreated conditions during pregnancy. A detailed review of the pregnancy-induced changes in drug metabolism, renal filtration, and active drug transport, which are being used by pharmacists to modify drug therapy during pregnancy can be found elsewhere. ${ }^{75}$

\subsection{3 | Ambulatory care PRN}

Given the long-term patient provider relationship, ambulatory care pharmacists are well positioned to broadly apply precision medicine approaches described in previous sections to their patients as part of existing comprehensive medication management services. Common precision medicine approaches already utilized by ambulatory care pharmacists include statin selection based on individual cardiovascular risk, warfarin dosing individualized to vitamin $\mathrm{K}$ intake, and medication optimization to patient-specific socioeconomic and functional status changes.

Applying patient-specific data and pharmacogenomics prior to the use of many common medications such as anticoagulants and antidepressants has been shown to improve efficacy, safety, and/or therapeutic outcomes in targeted patient populations. ${ }^{76,77}$ Pharmacogenomic data can also be beneficial when used within an existing treatment regimen to guide medication dosage/selection of alternative treatment regimens in at-risk patients (eg, those experiencing adverse effects or lack of response to proven therapies). Ambulatory care pharmacists are increasingly applying the principles of precision medicine, particularly pharmacogenomics, across a range of practice models including pharmacist-led chronic disease state or medication management services, consultation services to help interpret pharmacogenomic test results, and standalone pharmacist-led pharmacogenomics clinics. ${ }^{78,79}$ When genomic data are available, ambulatory care pharmacists should utilize these data to help guide medication selection (ie, statin selection in familial hypercholesterolemia, chemoprevention regimen in BRCA 
gene carriers). As direct-to-consumer genetic testing expands, ambulatory care pharmacists will also increasingly be called upon from inside and outside the profession to educate patients on the merits of pharmacogenomic and direct-to-consumer genetic testing and offer guidance to providers on applying direct-to-consumer genetic test results to clinical decision making. ${ }^{80,81}$

\section{3 | Drug development and outcomes specialties}

\subsection{1 | Pharmaceutical industry}

Competition throughout the pharmaceutical industry in addition to government initiatives and acceleration of the drug approval process has fostered development of precision medicines that have changed the treatment landscape for many diseases. New products have been designed with a therapeutic advantage or less risk of toxicity to the standard of care for a condition (eg, ledipasvir/sofosbuvir for hepatitis C) while others are the first treatments for special populations (eg, nusinersen for spinal muscular atrophy). Drug discovery targeted at specific genetic features (biomarkers) has transformed the treatment of many types of cancers, as discussed in the oncology section. Products with selective targets may have an associated companion diagnostic test to confirm presence or expression of the biomarker prior to treatment. ${ }^{82}$ Many of the new products are classified as specialty medications and have higher medication costs per treatment to counterbalance use in a more specific population.

Gene therapy is an innovative technology developed to treat or cure disease by incorporating new genes, replacing mutant genes, or silencing mutated genes. ${ }^{83}$ Two gene therapies are FDA-approved for the treatment of specific types of cancer in which a custom product is created for each patient by extracting the patient's own T-cells and integrating a new gene. ${ }^{84,85}$ Following genetic modification, the Tcells express a receptor that can recognize and attack malignant cells. The first viral vector gene therapy was FDA-approved to replace a mutated gene associated with vision loss and blindness with a normal gene by intraocular injection. ${ }^{86}$ Gene therapies with the ability to silence mutated genes are still under development. The recent approval of the antisense oligonucleotide nusinersen for the treatment of spinal muscular atrophy (SMA) is a milestone for both SMA treatment and the clinical availability of oligonucleotide technologies. $^{87}$

As the concept of precision medicine continues to evolve, designs of clinical trials are also advancing. ${ }^{88}$ Drug development in oncology has been on the forefront of implementing new clinical trial designs due to the discovery of specific biomarkers, availability of expedited review from the regulatory bodies, and better return on investment compared with other disease states. ${ }^{89}$ For example, early phase drug development in oncology frequently employs umbrella and basket trial designs. ${ }^{90}$ Basket trial design involves screening patients with different oncologic diseases or histological features for a specific drug target before enrolling only target-positive patients into a trial. ${ }^{91}$ Umbrella trial design focuses on screening multiple patients for various biomarkers and then stratifying all screened patients into drug treatment arms based on the presence of specific biomarkers. Such designs result in smaller patient enrollments per study drug. Smaller clinical trials will become more prevalent compared with the traditional study designs with subgroup analyses. ${ }^{88}$ However, several drawbacks and roadblocks limit widespread implementation of the new trial designs. Companion diagnostics are necessary for identifying and enrolling patients with the right targets into clinical trials, but only a small portion of agents with companion diagnostics reach late-stage drug development. ${ }^{89,90}$ New trial designs are also at a disadvantage due to small numbers of enrolled patients, insufficient safety data from small sample sizes, lack of outcomes data in negative-target patients, and unclear fit into the current regulatory guidelines. ${ }^{90}$ Nonetheless, different players in the health care industry ranging from pharmaceutical industry to health care providers will need to create systems and improve these developing trial designs to allow new targeted agents to reach the right patient at the right time, which is at the core of precision medicine.

\subsection{2 | Health outcomes}

Value can be defined in various ways. Economic value is what resources, time, money, or lost opportunities an individual is willing to spend to receive a good or service. In health care, value is defined as an outcome-clinical, patient satisfaction, quality of life-compared with a cost and is usually determined through cost-effectiveness and cost-utility analyses. Value must be evident for universal acceptance of health care services and recommendations by providers, patients, and payers. When applied, precision medicine may lead to selection of efficacious medications, avoidance of subtherapeutic drugs, and reduction of adverse events, adverse drug interactions, and emergency room visits. However, data showing improved clinical and economic outcomes with genotype-guided therapy are currently limited. Additional data are expected from on-going trials.

Demonstrating value in precision medicine presents multiple challenges. Value is determined on a population basis; however, precision medicine affects subsets of patients and even individuals. Additionally, testing costs significantly influence the value of personalized treatments and may not always be cost-effective. ${ }^{92}$ The limited data from pragmatic studies with long-term outcomes further complicates value analysis. ${ }^{93}$ Ongoing trials, adoption of electronic health records (EHR), and the ability to generate "big data" may address some barriers. However, this may result in changes to outcome assessment, require new models to assign value, and even generate new definitions of value. Value may exist in hope, reduced uncertainty, potential to improve or prolong life, or scientific knowledge gains. ${ }^{94}$

There are mixed opinions on whether data from randomized controlled trials, the evidence gold standard, are necessary to support adoption of genotype-guided therapy in practice. Some argue that, as with everything in health care, precision medicine must demonstrate the value of the care provided. However, demonstrating positive clinical outcomes and cost effectiveness with each example of genotypeguided therapy will likely be a slow process, especially if a randomized controlled trial is required for each example. Others believe that 
genotype is yet another factor to assist with drug selection and dosing, much like serum creatinine. Indeed, serum creatinine is routinely measured prior to drug prescribing, yet there are no randomized controlled trials demonstrating its benefit. Regardless, the emergence of additional data and ongoing real-world experiences will continue to help define the value of precision medicine.

\section{3 | CONCLUSION}

Pharmacists have long utilized individual patient factors and considerations for medication optimization. As medication experts committed to comprehensive medication management, they are well positioned to ensure each patient receives the right dose of the right medicine at the right time. In today's age of "precision medicine," clinical pharmacy practice has grown to incorporate genotype and gene expression data along with more traditional patient specific characteristics to better tailor drug therapy. As pharmacists generations ago learned to embrace and leverage therapeutic drug monitoring to improve patient care and outcomes, today's clinical pharmacy practitioners are best positioned to apply genomic advances to inform selection of medication therapy.

Evidence-based clinical guidelines are available to direct medication use in the presence of pharmacogenomics information. As supporting evidence for clinical implementation of pharmacogenomics is generated and pharmacogenomic test results become increasingly available, pharmacists should prepare to lead the adoption of this information into routine patient care as they have traditionally done for therapeutic drug monitoring. Barriers to successful implementation of pharmacogenomics programs, as well as potential solutions, have been previously described in detail. ${ }^{95-98} \mathrm{~A}$ key challenge is incorporating genetic data appropriately with other clinical factors to optimize therapy and building clinical decision support to facilitate this in practice. We also need to move past reactive, single gene tests and move towards array-based, preemptive pharmacogenomic testing, as this is a more cost-effective approach. Supporting such a program, however, requires robust informatics support. There are a number of other urgent professional needs in this expanding area to support the development and sustainability of pharmacy practice models that appropriately incorporate pharmacist expertise, including developing avenues for consistent reimbursement of pharmacist cognitive services, integration of genomic information into existing pharmacy practice models, consistent documentation and access to this information, and large-scale pharmacist and clinician educational efforts. Education is critical to ensure that clinicians are aware of clinically significant pharmacogenomic associations and the availability of testing. The ability as a profession to meet these needs will influence whether pharmacists are able to scale precision medicine services into more widespread and sustainable clinical implementation models.

\section{CONFLICTS OF INTEREST}

The authors declare no conflicts of interest.

\section{ORCID}

Kelly E. Caudle (D) https://orcid.org/0000-0002-5663-3793

Roseann S. Gammal (iD) https://orcid.org/0000-0001-7550-042X

Erin F. Barreto (D) https://orcid.org/0000-0002-0996-1487

Shubha Bhat (D) https://orcid.org/0000-0002-7023-9506

Christopher R. Ensor (D) https://orcid.org/0000-0003-0990-1406

Stephanie A. Flowers (D) https://orcid.org/0000-0001-5149-9444

Rena A. Gosser (iD https://orcid.org/0000-0002-7362-9219

Lamis R. Karaoui (D) https://orcid.org/0000-0002-7857-7374

Jonathan G. Leung (D) https://orcid.org/0000-0003-3836-9375

Angela Q. Maldonado (D) https://orcid.org/0000-0002-9633-1247

Michael A. Smith (D) https://orcid.org/0000-0002-9762-0104

\section{REFERENCES}

1. NIH. What is precision medicine? [cited 2018 Sept 4]. Available from: https://ghr.nlm.nih.gov/primer/precisionmedicine/definition.

2. Bishop JR, Ellingrod VL. Precision pharmacotherapy enables precision medicine. Pharmacotherapy. 2017;37(9):985-987.

3. Khanna D, Fitzgerald JD, Khanna PP, et al. American College of Rheumatology guidelines for management of gout. Part 1: Systematic nonpharmacologic and pharmacologic therapeutic approaches to hyperuricemia. Arthritis Care Res (Hoboken). 2012, 2012;64(10): 1431-1446.

4. CPIC. Clinical Pharmacogenetics Implementation Consortium guidelines [cited 2018 Dec 5]. Available from: https://cpicpgx.org/ guidelines/.

5. Ross CJ, Visscher H, Sistonen J, et al., CPNDS Consortium. The Canadian Pharmacogenomics Network for Drug Safety: A model for safety pharmacology. Thyroid. 2010;20(7):681-687.

6. Swen JJ, Nijenhuis $M$, de Boer $A$, et al. Pharmacogenetics: From bench to byte--an update of guidelines. Clin Pharmacol Ther. 2011;89 (5):662-673.

7. Etienne-Grimaldi MC, Boyer JC, Thomas F, et al., Collective work by Groupe de Pharmacologie Clinique Oncologique (GPCO-Unicancer), French Réseau National de Pharmacogénétique Hospitaliére (RNPGx). UGT1A1 genotype and irinotecan therapy: General review and implementation in routine practice. Fundam Clin Pharmacol. 2015;29(3): 219-237.

8. Clarke G, Stilling RM, Kennedy PJ, Stanton C, Cryan JF, Dinan TG. Minireview: Gut microbiota: The neglected endocrine organ. Mol Endocrinol. 2014;28(8):1221-1238.

9. Whelton PK, Carey RM, Aronow WS, et al. 2017 ACC/AHA/ AAPA/ABC/ACPM/AGS/APhA/ASH/ASPC/NMA/PCNA Guideline for the prevention, detection, evaluation, and management of high blood pressure in adults: A report of the American College of Cardiology/American Heart Association Task Force on clinical practice guidelines. Hypertension. 2018;71(6):1269-1324.

10. Bloche MG. Race-based therapeutics. N Engl J Med. 2004;351(20): 2035-2037.

11. Yeh RW, Secemsky EA, Kereiakes DJ, et al., for the DAPT Study Investigators. Development and validation of a prediction rule for benefit and harm of dual antiplatelet therapy beyond 1 year after percutaneous coronary intervention. JAMA. 2016;315(16):1735-1749.

12. Rao SV, McCoy LA, Spertus JA, et al. An updated bleeding model to predict the risk of post-procedure bleeding among patients undergoing percutaneous coronary intervention: A report using an expanded bleeding definition from the National Cardiovascular Data Registry CathPCI Registry. JACC Cardiovasc Interv. 2013;6(9):897-904. 
13. Witt DM. Approaches to optimal dosing of vitamin $\mathrm{K}$ antagonists. Semin Thromb Hemost. 2012;38(7):667-672.

14. Dong AN, Tan BH, Pan Y, Ong CE. Cytochrome P450 genotypeguided drug therapies: An update on current states. Clin Exp Pharmacol Physiol. 2018;45(10):991-1001.

15. Cavallari LH, Pratt VM. Building evidence for clinical use of pharmacogenomics and reimbursement for testing. Adv Mol Pathol. 2018;1 (1):125-134.

16. Tighe SK, Mahon PB, Potash JB. Predictors of lithium response in bipolar disorder. Ther Adv Chronic Dis. 2011;2(3):209-226.

17. Hiemke C, Bergemann N, Clement HW, et al. Consensus guidelines for therapeutic drug monitoring in neuropsychopharmacology: Update 2017. Pharmacopsychiatry. 2018;51(1-02):e1.

18. Rosenblat JD, Lee $Y$, Mclntyre RS. Does pharmacogenomic testing improve clinical outcomes for major depressive disorder? A systematic review of clinical trials and cost-effectiveness studies. J Clin Psychiatry. 2017;78(6):720-729.

19. Rahman T, Ash DM, Lauriello J, Rawlani R. Misleading guidance from pharmacogenomic testing. Am J Psychiatry. 2017;174(10):922-924.

20. Roberts JA, Lipman J. Pharmacokinetic issues for antibiotics in the critically ill patient. Crit Care Med. 2009;37(3):840-851. quiz 59.

21. Seymour $\mathrm{CW}$, Gomez $\mathrm{H}$, Chang $\mathrm{CH}$, et al. Precision medicine for all? Challenges and opportunities for a precision medicine approach to critical illness. Crit Care. 2017;21(1):257.

22. Wong HR, Cvijanovich NZ, Anas N, et al. Pediatric Sepsis Biomarker Risk Model-II: Redefining the Pediatric Sepsis Biomarker Risk Model With Septic Shock Phenotype. Crit Care Med. 2016 Nov;44(11): 2010-2017.

23. Calfee CS, Delucchi K, Parsons PE, et al. Subphenotypes in acute respiratory distress syndrome: Latent class analysis of data from two randomised controlled trials. Lancet Respir Med. 2014;2(8):611-620.

24. Empey PE. Genetic predisposition to adverse drug reactions in the intensive care unit. Crit Care Med. 2010;38(6 Suppl):S106-S116.

25. Skyler JS, Bakris GL, Bonifacio E, et al. Differentiation of diabetes by pathophysiology, natural history, and prognosis. Diabetes. 2017;66(2): 241-255.

26. Florez JC. Pharmacogenetics in type 2 diabetes: Precision medicine or discovery tool? Diabetologia. 2017;60(5):800-807.

27. Sandborn WJ. Crohn's disease evaluation and treatment: Clinical decision tool. Gastroenterology. 2014;147(3):702-705.

28. AASLD-IDSA. Recommendations for testing, managing, and treating hepatitis C [cited 2018 April 1]. Available from: www. hcvguidelines.org.

29. El Rouby N, Lima JJ, Johnson JA. Proton pump inhibitors: From CYP2C19 pharmacogenetics to precision medicine. Expert Opin Drug Metab Toxicol. 2018;14(4):447-460.

30. Walko C, Kiel PJ, Kolesar J. Precision medicine in oncology: New practice models and roles for oncology pharmacists. Am J Health Syst Pharm. 2016;73(23):1935-1942.

31. Vogelstein B, Papadopoulos N, Velculescu VE, Zhou S, Diaz LA Jr, Kinzler KW. Cancer genome landscapes. Science (New York, NY). 2013;339(6127):1546-1558.

32. Flaherty $\mathrm{KT}$, Infante JR, Daud A, et al. Combined BRAF and MEK inhibition in melanoma with BRAF V600 mutations. N Engl J Med. 2012; 367(18):1694-1703.

33. Knepper TC, Bell GC, Hicks JK, et al. Key lessons learned from Moffitt's Molecular Tumor Board: The Clinical Genomics Action Committee experience. Oncologist. 2017;22(2):144-151.

34. Relling MV, Gardner EE, Sandborn WJ, et al., Clinical Pharmacogenetics Implementation Consortium. Clinical pharmacogenetics implementation consortium guidelines for thiopurine methyltransferase genotype and thiopurine dosing: 2013 update. Clin Pharmacol Ther. 2013; 93(4):324-325

35. Amstutz U, Henricks LM, Offer SM, et al. Clinical Pharmacogenetics Implementation Consortium (CPIC) guideline for dihydropyrimidine dehydrogenase genotype and fluoropyrimidine dosing: 2017 update. Clin Pharmacol Ther. 2018;103(2):210-216.

36. Henricks LM, Lunenburg C, de Man FM, et al. DPYD genotype-guided dose individualisation of fluoropyrimidine therapy in patients with cancer: A prospective safety analysis. Lancet Oncol. 2018;19(11): 1459-1467.

37. Department of Health and Human Services Panel on Antiretroviral Guidelines for Adults and Adolescents. Guidelines for the use of antiretroviral agents in adults and adolescents living with HIV [cited 2018 April 11]. Available from: http://aidsinfo.nih.gov/contentfiles/ Ivguidelines/AdultandAdolescentGL.pdf.

38. CDC. HIV and Older Americans [cited 2018 April 11]. Available from: www.cdc.gov/hiv/pdf/group/age/olderamericans/cdc-hiv-olderamericans.pdf.

39. Gammal RS, Court MH, Haidar CE, et al. Clinical Pharmacogenetics Implementation Consortium (CPIC) guideline for UGT1A1 and atazanavir prescribing. Clin Pharmacol Ther. 2016;99(4):363-369.

40. Vo TT, Varghese GS. Role of cytochrome P450 2B6 pharmacogenomics in determining efavirenz-mediated central nervous system toxicity, treatment outcomes, and dosage adjustments in patients with human immunodeficiency virus infection. Pharmacotherapy. 2016;36(12):1245-1254

41. Birdwell KA, Decker B, Barbarino JM, et al. Clinical Pharmacogenetics Implementation Consortium (CPIC) guidelines for CYP3A5 genotype and tacrolimus dosing. Clin Pharmacol Ther. 2015;98(1):19-24.

42. Barlam TF, Cosgrove SE, Abbo LM, et al. Implementing an antibiotic stewardship program: Guidelines by the Infectious Diseases Society of America and the Society for Healthcare Epidemiology of America. Clin Infect Dis. 2016;62(10):e51-e77.

43. Wenzler E, Timbrook TT, Wong JR, Hurst JM, MacVane SH. Implementation and optimization of molecular rapid diagnostic tests for bloodstream infections. Am J Health Syst Pharm. 2018;75(16): 1191-1202.

44. Moriyama B, Obeng AO, Barbarino J, et al. Clinical Pharmacogenetics Implementation Consortium (CPIC) guidelines for CYP2C19 and voriconazole therapy. Clin Pharmacol Ther. 2017;102(1):45-51.

45. Hocher B, Adamski J. Metabolomics for clinical use and research in chronic kidney disease. Nat Rev Nephrol. 2017;13(5):269-284.

46. Yokoyama H, Narita I, Sugiyama H, et al. Drug-induced kidney disease: A study of the Japan Renal Biopsy Registry from 2007 to 2015. Clin Exp Nephrol. 2016;20(5):720-730.

47. Stockbridge CLANL. Biomarker qualification determination. FDA. 2018. Available from: https://www.fda.gov/downloads/Drugs/ DevelopmentApprovalProcess/ DrugDevelopmentToolsQualificationProgram/ BiomarkerQualificationProgram/UCM618888.pdf.

48. Solomon R, Goldstein S. Real-time measurement of glomerular filtration rate. Curr Opin Crit Care. 2017;23(6):470-474.

49. Basu RK, Gist K, Wheeler DS. Improving acute kidney injury diagnostics using predictive analytics. Curr Opin Crit Care. 2015;21(6) 473-478.

50. Dansie EJ, Turk DC. Assessment of patients with chronic pain. $\mathrm{Br} J$ Anaesth. 2013;111(1):19-25.

51. Owusu Obeng A, Hamadeh I, Smith M. Review of opioid pharmacogenetics and considerations for pain management. Pharmacotherapy. 2017;37(9):1105-1121.

52. Hicks JK, Sangkuhl K, Swen JJ, et al. Clinical pharmacogenetics implementation consortium guideline (CPIC) for CYP2D6 and CYP2C19 genotypes and dosing of tricyclic antidepressants: 2016 update. Clin Pharmacol Ther. 2017;102(1):37-44.

53. Phillips EJ, Sukasem C, Whirl-Carrillo $M$, et al. Clinical pharmacogenetics implementation consortium guideline for HLA genotype and use of carbamazepine and oxcarbazepine: 2017 update. Clin Pharmacol Ther. 2018;103(4):574-581. 
54. Ting S, Schug S. The pharmacogenomics of pain management: Prospects for personalized medicine. J Pain Res. 2016;9:49-56.

55. Fleisher LA, Fleischmann KE, Auerbach AD, et al., American College of Cardiology, American Heart Association. 2014 ACC/AHA guideline on perioperative cardiovascular evaluation and management of patients undergoing noncardiac surgery: A report of the American College of Cardiology/American Heart Association Task Force on practice guidelines. J Am Coll Cardiol. 2014;64(22):e77-e137.

56. Futier E, Lefrant JY, Guinot PG, et al., for the INPRESS Study Group. Effect of individualized vs standard blood pressure management strategies on postoperative organ dysfunction among high-risk patients undergoing major surgery: A randomized clinical trial. JAMA. 2017;318(14):1346-1357.

57. Manning MW, Dunkman WJ, Miller TE. Perioperative fluid and hemodynamic management within an enhanced recovery pathway. J Surg Oncol. 2017;116(5):592-600.

58. Hopkins PM, Ruffert H, Snoeck MM, et al. European Malignant Hyperthermia Group guidelines for investigation of malignant hyperthermia susceptibility. Br J Anaesth. 2015;115(4):531-539.

59. Gan TJ, Diemunsch P, Habib AS, et al., Society for Ambulatory Anesthesia. Consensus guidelines for the management of postoperative nausea and vomiting. Anesth Analg. 2014;118(1):85-113.

60. Bratzler DW, Dellinger EP, Olsen KM, et al., American Society of Health-System Pharmacists (ASHP), Infectious Diseases Society of America (IDSA), Surgical Infection Society (SIS), Society for Healthcare Epidemiology of America (SHEA). Clinical practice guidelines for antimicrobial prophylaxis in surgery. Surg Infect (Larchmt). 2013;14(1):73-156.

61. Clancy JP, Johnson SG, Yee SW, et al. Clinical Pharmacogenetics Implementation Consortium (CPIC) guidelines for ivacaftor therapy in the context of CFTR genotype. Clin Pharmacol Ther. 2014;95(6): 592-597.

62. Kraft M. Asthma phenotypes and interleukin-13--moving closer to personalized medicine. N Engl J Med. 2011;365(12):1141-1144.

63. Fajt ML, Wenzel SE. Asthma phenotypes and the use of biologic medications in asthma and allergic disease: The next steps toward personalized care. J Allergy Clin Immunol. 2015;135(2):299-310. quiz 1.

64. Bartelink IH, Rademaker CM, Schobben AF, van den Anker JN. Guidelines on paediatric dosing on the basis of developmental physiology and pharmacokinetic considerations. Clin Pharmacokinet. 2006;45(11): 1077-1097.

65. Momper JD, Wagner JA. Therapeutic drug monitoring as a component of personalized medicine: Applications in pediatric drug development. Clin PharmacolTher. 2014;95(2):138-140.

66. Young TE. Therapeutic drug monitoring--the appropriate use of drug level measurement in the care of the neonate. Clin Perinatol. 2012;39 (1):25-31.

67. Gammal RS, Crews KR, Haidar CE, et al. Pharmacogenetics for safe codeine use in sickle cell disease. Pediatrics. 2016;138(1):1-12.

68. Hawcutt DB, Cooney L, Oni L, Pirmohamad M. Precision dosing in children. Expert Rev Precis Med Drug Dev. 2016;1(1):69-78.

69. Wang WT, James SK, Wang TY. A review of sex-specific benefits and risks of antithrombotic therapy in acute coronary syndrome. Eur Heart J. 2017;38(3):165-171.

70. Mosca L, Benjamin EJ, Berra K, et al. Effectiveness-based guidelines for the prevention of cardiovascular disease in women--2011 update: A guideline from the american heart association. Circulation. 2011; 123(11):1243-1262.

71. Seeman MV. Secondary effects of antipsychotics: Women at greater risk than men. Schizophr Bull. 2009;35(5):937-948.

72. Seeman MV. Gender differences in the prescribing of antipsychotic drugs. Am J Psychiatry. 2004;161(8):1324-1333.

73. Bano S, Akhter S, Afridi MI. Gender based response to fluoxetine hydrochloride medication in endogenous depression. J Coll Physicians Surg Pak. 2004;14(3):161-165.
74. Kornstein SG, Schatzberg AF, Thase ME, et al. Gender differences in treatment response to sertraline versus imipramine in chronic depression. Am J Psychiatry. 2000;157(9):1445-1452.

75. Tasnif $\mathrm{Y}$, Morado J, Hebert MF. Pregnancy-related pharmacokinetic changes. Clin Pharmacol Ther. 2016;100(1):53-62.

76. Bradley P, Shiekh M, Mehra V, et al. Improved efficacy with targeted pharmacogenetic-guided treatment of patients with depression and anxiety: A randomized clinical trial demonstrating clinical utility. J Psychiatr Res. 2018;96:100-107.

77. Gage BF, Bass AR, Lin H, et al. Effect of genotype-guided warfarin dosing on clinical events and anticoagulation control among patients undergoing hip or knee arthroplasty: The GIFT randomized clinical trial. JAMA. 2017;318(12):1115-1124.

78. Dunnenberger HM, Biszewski M, Bell GC, et al. Implementation of a multidisciplinary pharmacogenomics clinic in a community health system. Am J Health Syst Pharm. 2016;73(23):1956-1966.

79. Ferreri SP, Greco AJ, Michaels NM, et al. Implementation of a pharmacogenomics service in a community pharmacy. J Am Pharm Assoc. 2014;54(2):172-180.

80. Mills R, Haga SB. Clinical delivery of pharmacogenetic testing services: A proposed partnership between genetic counselors and pharmacists. Pharmacogenomics. 2013;14(8):957-968.

81. Ramos E, Weissman SM. The dawn of consumer-directed testing. Am J Med Genet C Semin Med Genet. 2018;178(1):89-97.

82. U.S. Food \& Drug Administration. List of cleared or approved companion diagnostic devices (in vitro and imaging tools). 2018 [cited 2018 April 20]. Available from: www.fda.gov/MedicalDevices/ ProductsandMedicalProcedures/InVitroDiagnostics/ucm301431.htm.

83. U.S. Food \& Drug Adminstration. What is gene therapy? How does it work? 2017 [cited 2018 April 20]. Available from: www.fda.gov/ ForConsumers/ConsumerUpdates/ucm589197.htm.

84. U.S. Food \& Drug Administration. FDA approval brings first gene therapy to the United States. 2018 [cited 2018 April 20]. Available from: www.fda.gov/NewsEvents/Newsroom/PressAnnouncements/ ucm574058.htm.

85. U.S. Food \& Drug Administration. FDA approves CAR-T cell therapy to treat adults with certain types of large B-cell lymphoma. 2018 [cited 2018 April 20]. Available from: www.fda.gov/NewsEvents/ Newsroom/PressAnnouncements/ucm581216.htm.

86. U.S. Food \& Drug Administration. FDA approves novel gene therapy to treat patients with a rare form of inherited vision loss. 2018 [cited 2018 April 20]. Available from: www.fda.gov/NewsEvents/ Newsroom/PressAnnouncements/UCM589467.htm?utm_campaign= 12192017_PR_FDA\%20approves\%20first\%20gene\%20therapy\% 20treatment\&utm_medium=email\&utm_source=Eloqua.

87. Finkel RS, Mercuri E, Darras BT, et al. Nusinersen versus sham control in infantile-onset spinal muscular atrophy. N Engl J Med. 2017;377 (18):1723-1732.

88. Eichler HG, Sweeney F. The evolution of clinical trials: Can we address the challenges of the future? Clin Trials. 2018;15(1_suppl): 27-32.

89. Milne C-P, Cohen JP, Chakravarthy R. Where is personalized medicine in industry heading? Nat Rev Drug Discov. 2015;14:812-813.

90. Hollingsworth SJ, Biankin AV. The challenges of precision oncology drug development and implementation. Public Health Genomics. 2015; 18(6):338-348.

91. Woodcock J, LaVange LM. Master protocols to study multiple therapies, multiple diseases, or both. N Engl J Med. 2017;377(1):62-70.

92. Verbelen M, Weale ME, Lewis CM. Cost-effectiveness of pharmacogenetic-guided treatment: Are we there yet? Pharmacogenomics J. 2017;17(5):395-402.

93. Chen Y, Guzauskas GF, Gu C, et al. Precision health economics and outcomes research to support precision medicine: Big data meets patient heterogeneity on the road to value. J Pers Med. 2016; 6(4):1-8. 
94. Garrison LP Jr, Kamal-Bahl S, Towse A. Toward a broader concept of value: Identifying and defining elements for an expanded costeffectiveness analysis. Value Health. 2017;20(2):213-216.

95. Sperber NR, Carpenter JS, Cavallari LH, et al. Challenges and strategies for implementing genomic services in diverse settings: Experiences from the Implementing GeNomics In pracTicE (IGNITE) network. BMC Med Genomics. 2017;10(1):35.

96. Pritchard DE, Moeckel F, Villa MS, Housman LT, McCarty CA, McLeod HL. Strategies for integrating personalized medicine into healthcare practice. Per Med. 2017;14(2):141-152.

97. Arwood MJ, Chumnumwat S, Cavallari LH, Nutescu EA, Duarte JD. Implementing pharmacogenomics at your institution: Establishment and overcoming implementation challenges. Clin Transl Sci. 2016;9(5): 233-245.
98. Shuldiner AR, Relling MV, Peterson JF, et al., Pharmacogenomics Research Network Translational Pharmacogenetics Program Group. The pharmacogenomics research network translational pharmacogenetics program: Overcoming challenges of real-world implementation. Clin Pharmacol Ther. 2013;94(2):207-210.

How to cite this article: Caudle KE, Gammal RS, Karnes JH, et al. PRN OPINION PAPER: Application of precision medicine across pharmacy specialty areas. J Am Coll Clin Pharm. 2019;2: 288-302. https://doi.org/10.1002/jac5.1107 